\title{
A Digitally Assisted Analog Cancellation System at RF Frequencies for Improving the Isolation Performance of a Ceramic Duplexer
}

\author{
Han Su, Ziming Wang, Sarah Ibrahim, Ronan Farrell \\ Department of Electronic Engineering, \\ National University of Ireland, Maynooth \\ Email: hsu@eeng.nuim.ie
}

\begin{abstract}
In order to reduce the isolation performance requirement of a duplexer in an FDD transceiver, this paper presents a TX leakage cancellation technique that is digitally controlled, operates at RF frequencies and is designed to suppress the in-band leakage with respect to the $\mathrm{RX}$ band. Not only the attenuation and phase, but also the delays are optimized by using the proposed MADF-DC estimation technique. In order to demonstrate the concept, the cancellation system with a ceramic duplexer is prototyped using dedicated components for the transceiver test bench that uses a ceramic duplexer. The design bandwidth of the system is $35 \mathrm{MHz}$ at the centre frequency of 897.5 MHz. More than $11 \mathrm{~dB}$ and $20 \mathrm{~dB}$ of cancellation have been achieved by measurement and simulation, respectively.

Index Terms-Leakage, Duplexer, Cancellation, Interference
\end{abstract}

\section{INTRODUCTION}

A duplexer is a passive device that is used to separate the transmitter (TX) and the receiver (RX) in an FDD base station. Due to the limited isolation performance of the duplexer between TX and RX, a small portion of the signal at the output of the high power amplifier appears at RX. Such signal, known as the TX leakage, can be separated as the in-band and out-of-band portions with respect to the RX frequency band. The in-band leakage is mainly caused by the noise and intermodulation distortions of the high power amplifier (PA). It is more problematic as it falls within the same band as the received signal and can't be filtered using conventional filtering techniques. The existence of large in-band leakage causes the desensitization and saturation of the RF front-end.

A duplexer with high isolation performance between TX and $\mathrm{RX}$ is usually deployed in a base station in order to suppress the TX leakage and to avoid the saturation and the desensitization of the RX. However, such duplexer is expensive and bulky which makes them less favourable in a base station that uses the remote radio head (RRH) technology. More importantly, as wider bandwidth transmission and reception are desired, designing a duplexer with high isolation performance and limited cost and form factor is getting more difficult. In order to solve the problems that are mentioned above, the high isolation performance requirement of a duplexer needs to be relaxed. The additional TX leakage that is due to the replacement of a high performance duplexer with a lower one thus needs to be suppressed. This is an interesting topic that attracts the attentions of the researchers from both academia and industry.

In [1], a sub-band digital cancellation has been proposed with over $15 \mathrm{~dB}$ of cancellation for more than $100 \mathrm{MHz}$ bandwidth at baseband. Despite the outstanding leakage cancellation performance at the baseband using this technique, digital cancellations are incapable of solving the saturation problem.

A tap-based RF analog cancellation architecture has been proposed in [2] and is designed for suppressing the selfinterference in a same channel full duplex (SC-FD) system which uses a circulator or a configuration of separated antennas for basic isolation between TX and RX. As the characteristics of the isolation path of the configuration using either a circulator or antenna separation are significantly simpler than the ones of an off-the-shelf duplexer, great cancellation cannot be guaranteed when such technique is implemented in an FDD transceiver. Additionally, in this technique, no optimization techniques of the delays have been suggested, which further reduce the chances of successful cancellation using this technique in an FDD transceiver.

In [3], an analog cancellation architecture named as four port canceller (FPC) has been proposed for an FDD transceiver with a film bulk acoustic resonator (FBAR) duplexer. Using this technique, the amplitude and the phase of the replicated TX signal are varied by changing the values of the three capacitors in the RF cancellation block. Inherently, the cancellation bandwidth of this technique is limited to $5 \mathrm{MHz}$ for WCDMA band I.

In this paper, we propose a wideband TX leakage cancellation system for an FDD base station using an off-the-shelf duplexer with complex characteristics. Such system is tap-based and has the ability of not only adapting the amplitudes and phases, but also determining the optimal delays. Comparing to the published technique in [4], the cancellation architecture in this paper is more flexible and estimation technique is more robust and accurate. Furthermore, both simulation and measurement results have been presented in this paper.

\section{The Proposed Cancellation Architecture}

The block diagram of the proposed cancellation system is shown in Fig. 1. The system takes the advantage that the 
characteristics of the duplexer vary insignificantly over time and performs the estimation in off-line mode with the antenna and the cancellation block being turned off. Small change in the characteristics of the duplexer can be compensated online by adapting the attenuators and phase shifters, upon the completion of the modelling and cancellation. At the beginning of the estimation, the received signal before the low noise amplifier (LNA) is the leakage signal only, i.e., $y(t)=l(t)$ and is frequency down-converted to baseband to obtain $\hat{y}(n)$. The estimation of the duplexer is thus initialized by the reference, $\hat{x}(n)$ and the leakage $\hat{y}(n)$ at the baseband.

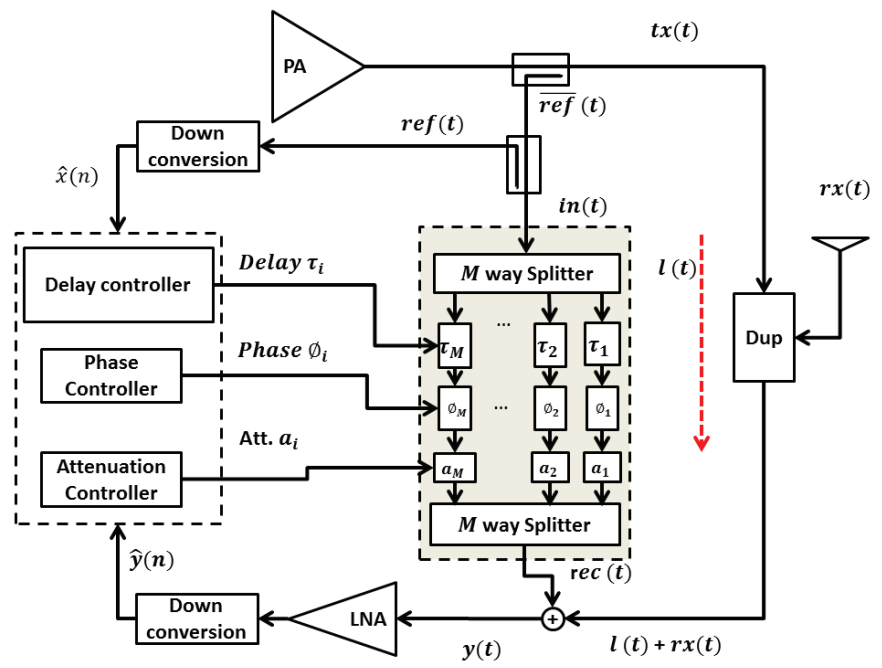

Fig. 1. The block diagram of the proposed cancellation system

A DSP unit is used to estimate the delays and tap weights with the baseband reference and leakage signals. In the case that tunable delay lines are deployed in the system, they, along with variable attenuators and phase shifters, can be tuned at the time of estimation. Otherwise, fixed delay lines are designed and implemented upon the completion of the estimation. Then the RF attenuators and phase shifters are adapted so that the resulting leakage is minimized. In theory, the values of the delay lines and attenuators in the cancellation path directly relate to the estimated delays and the magnitudes of the adapted tap weights from the modelling, respectively. The value of a phase shifter in each cancellation path is the sum of two portions. One is the phase of the corresponding estimated tap weight. The other is the effective phase rotation of the local oscillating signal that is caused by the delay in the corresponding tap. This architecture can be considered as an analog implementation of a sparse FIR filter. Upon the completion of the modelling and cancellation, the antenna can be turned on. As the received signal is not correlated with the leakage, only the later one is cancelled without affecting the $\mathrm{RX}$ signal significantly.

\section{Estimation TechniQue}

Sparse system identification technique, such as the classic adaptive delay filter (C-ADF) [5], is preferred for the delay estimation in order to minimize the number of taps and at the same time, maximize the cancellation performance. This is especially true for a transceiver with a standard duplexer due to its complex characteristics within the band of interest. As the delay estimation accuracy of the C-ADF is limited to the critical sampling frequency at the baseband, the modified adaptive delay filter using direct cross-correlation (MADFDC) is proposed in this paper.

Similar to C-ADF, MADF-DC is a sequential operation technique due to the fact that the delay estimation of a later tap or stage depends on the previous ones. In order to achieve a better accuracy, three additional parameters, including the up-sampling ratio, minimum and maximum delay limits, are swept within a given range. A full estimation procedure is performed using each unique combination of these three parameters, until no new combinations are available. The estimated delays and the tap weights of the combination with which the sum of squares of the leakage is minimized are the final estimation results. The operation structure of the proposed MADF-DC for each set of the up-sampling ratio, maximum and minimum delay limits parameters is shown in Fig. 2.

MADF-DC uses the cross-correlation to approximate the impulse response of the isolation path of the duplexer. For each tap (stage), cross-correlation is performed using the upsampled reference and the previous error signals, i.e.,

$$
R_{\hat{x}_{u p} \hat{e} p_{u p}}=\hat{x}_{u p}\left(n_{u p}\right) \star \hat{e p}{ }_{u p}\left(n_{u p}\right)
$$

where the operator $\star$ represents cross-correlation, and $\hat{x}_{u p}\left(n_{u p}\right)=\operatorname{UpSample}\{\hat{x}(n)\}$ and $\hat{e p_{u p}}\left(n_{u p}\right)=$ UpSample $\{\hat{e p}(n)\}$ are the up-sampled signals of the critically sampled reference signal $\hat{x}(n)$ and the previous error signal $\hat{e p}(n)$, respectively. The sample indexes of the critically sampled and up-sampled signals are denoted as $n=1,2, \ldots, N$ and $n_{u p}=1,2, \ldots, N_{u p}$, respectively, where $N$ and $N_{u p}$ are the corresponding total number of samples. The delay at which the absolute value of the cross-correlation vector is maximized is chosen to be the estimated one of the current tap, providing that two following conditions are met. The first condition is that the estimated delay of the current tap has not been obtained during the previous estimations, providing that the current tap is not the first one. The delays are also bounded by the given maximum and minimum delay limits. The two conditions must be satisfied at the same time. Otherwise the delay of the next peak correlation value is chosen until the conditions are fully met. The mathematical representation is thus

$$
\begin{aligned}
\hat{d}_{m}=\underset{q}{\arg \max } & \left(\left|R_{\hat{x}_{u p}, \hat{e p}_{u p}}(q)\right|\right) \\
\text { subject to: } & \hat{d}_{m} \neq d_{i} \quad \text { for } m \geq 2, \\
& d_{\text {min }} \leq \hat{d}_{m} \leq d_{\text {max }}
\end{aligned}
$$

where $d_{\max }$ and $d_{\min }$ are the bounding limits of the delay, and the subscript $m=1,2, \ldots, M$ and $i=1,2, \ldots, m-1$ represent the indexes of the current and previous taps, respectively.

Once the delay of one stage is estimated, the coefficients of the taps of which the delays have been estimated are updated 


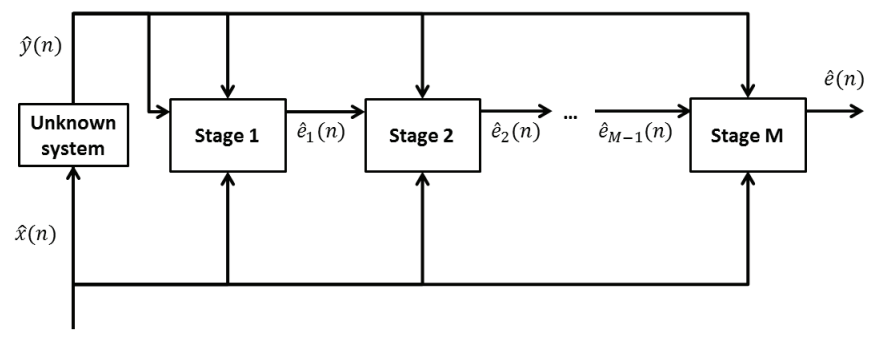

(a)

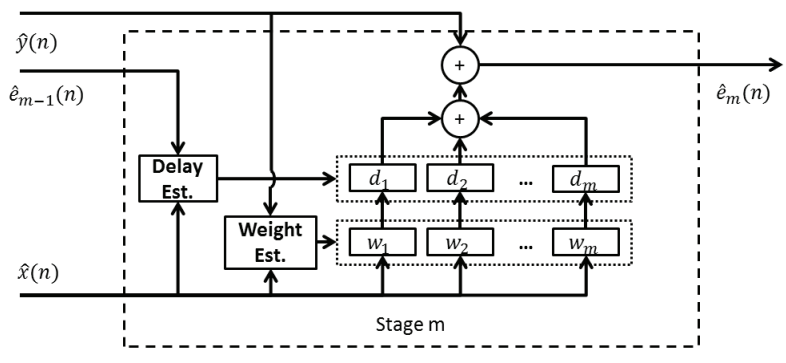

(b)

Fig. 2. The block diagram of the MADF-DC technique. (a) High level block diagram. (b) Block Diagram example of the $m^{t h}$ stage.

using LS algorithm under the critical sampling. Comparing to the classic technique, MADF-DC updates all the weights of estimated taps, instead of only the one of the current tap. The input matrix for the estimated $m$ taps is given by

$$
\hat{X}=\left[\hat{x}^{T}\left(n-d_{1}\right), \hat{x}^{T}\left(n-d_{2}\right), \ldots, \hat{x}^{T}\left(n-d_{m}\right)\right]
$$

where $\hat{x}(n)$ is the critically sampled reference signal, $d_{k}=\frac{\hat{d}_{k}}{K}$ is the estimated fractional delay of the corresponding tap at the baseband, $k=1,2, \ldots, m$ is the index of the estimated taps and $K$ is the up-sampling ratio.

\section{EXPERIMENTAL VALIDATION}

\section{A. Measurement Setup}

The measurement setup has been shown in Fig. 3. The components in the test bench are dedicated ones that are either purchased off-the-shelf or designed and fabricated in the laboratory. It should be emphasized that the measurement setup is only to demonstrate the concept of this paper. It is possible to shrink the entire system into an extremely small form factor, possibly in chip size. However, optimization of physical parameters such as the size of the test bench is outside the scope of this paper.

The duplexer under test is a ceramic type (SCD900AE4) that is designed for WCDMA band VIII and manufactured by Samnova. The testing signal is white Gaussian noise which is composed using MATLAB and generated by the vector signal generator (SMU) by Rohde \& Schwarz. The centre frequency and bandwidth of the testing signal are set to be $897.5 \mathrm{MHz}$ and $35 \mathrm{MHz}$, respectively. This signal is for training and modelling purposes. The white characteristics are only required for modelling. Upon the completion of modelling, the cancellation

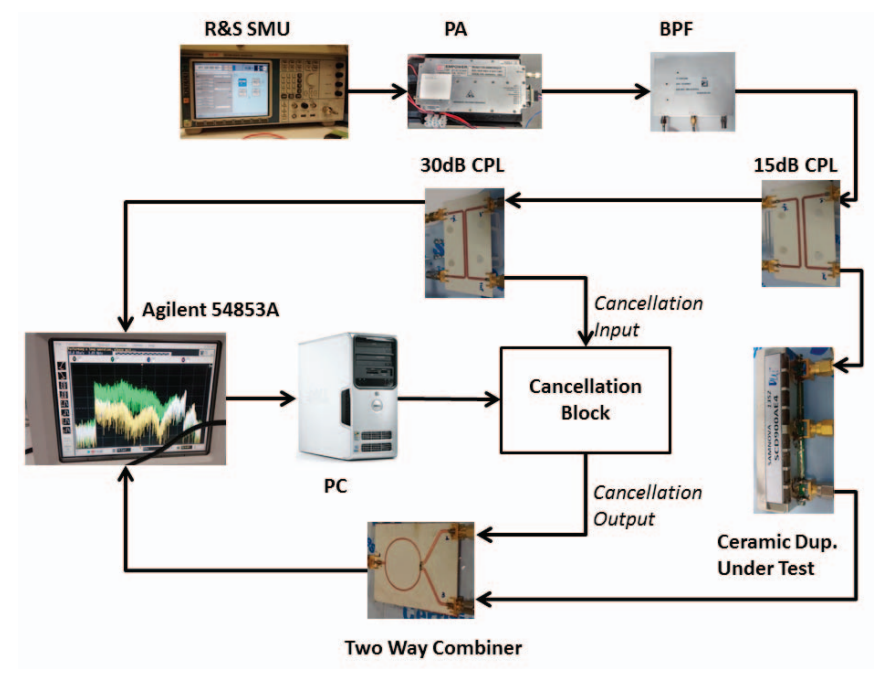

(a)

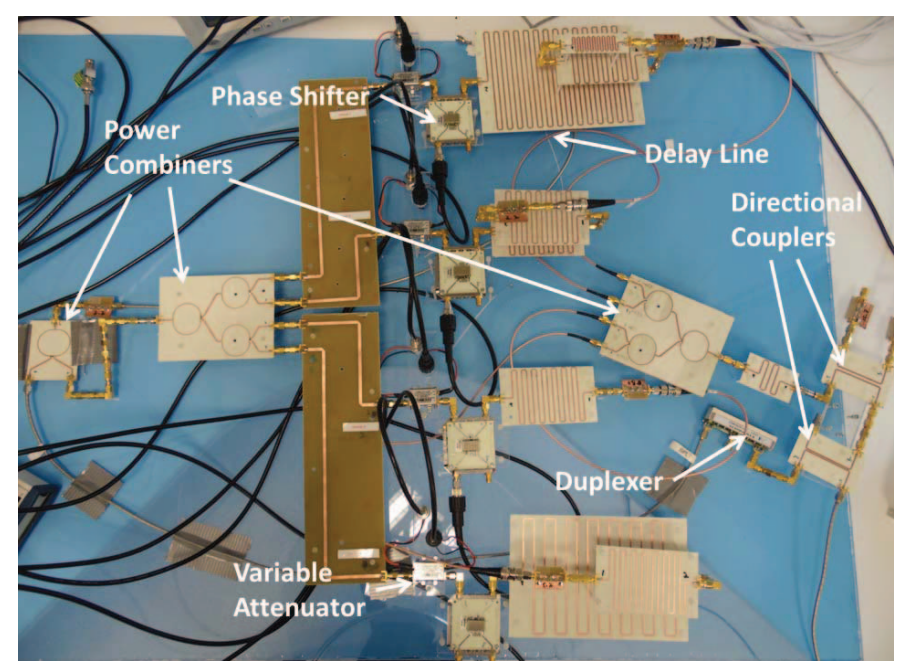

(b)

Fig. 3. The block diagrams of the measurement setup. (a) The overview of the setup. (b) The diagram of the cancellation block

performance is independent of signal types. In this bench, this signal is also for simulating the in-band (with respect to the RX band) TX leakage which is caused by the distortions and interferences of the PA in an actual transceiver within the RX band. The reference and the leakage signals are captured by the oscilloscope (54853A) from Agilent Technologies which is capable of sampling up to $20 \mathrm{GSa} / \mathrm{s}$ and has the bandwidth of $2.5 \mathrm{GHz}$. The bandpass filter is deployed in order to suppress the harmonic distortions that are caused by the PA (1184BBM3O6QCK from Empower RF systems).

The implemented cancellation block has been shown in Fig. 3b. There is a variable attenuator (ZX73-2500+ by Mini-Circuits), a $360^{\circ}$ phase shifter (SPHSA-152+ by MiniCircuits), a fixed attenuator and a fixed delay line in each of the four cancellation paths. The delay line of each cancellation path is constructed with a number of smaller delays, depending on the estimated value. The implementation error of the delays before adapting the variable components is no more than $0.5 \mathrm{~ns}$ 
from the estimated ones. Upon the completion of constructing the measurement test bench, the simulated annealing which is a global optimization technique has been used in order to adapt the variable components for the best cancellation performance.

\section{B. Measured Results}

Both measured and simulated leakages after cancellation are shown in Fig. 4, as well as the one before cancellation. As we are more concerning about the peak power within the frequency band of interest, a term, named as peakto-peak cancellation (P-P. Can.) performance is defined for the evaluation of the proposed cancellation system. The PP. cancellation performance is the difference between the peak powers in log scale before and after the cancellation within the band of interest. It can be seen that the P-P. performance of the measured result is approximately $11 \mathrm{~dB}$ for $35 \mathrm{MHz}$ bandwidth, whereas the simulated result shows that greater than $20 \mathrm{~dB}$ of P-P. cancellation can be achieved. In the case that the bandwidth is reduced to $30 \mathrm{MHz}$ at the same centre frequency, such simulated performance can be as large as approximately $30 \mathrm{~dB}$ using only four taps. The measured performance increases by approximately $5 \mathrm{~dB}$ for $30 \mathrm{MHz}$ bandwidth. Better performance can be achieved with additional number of taps.

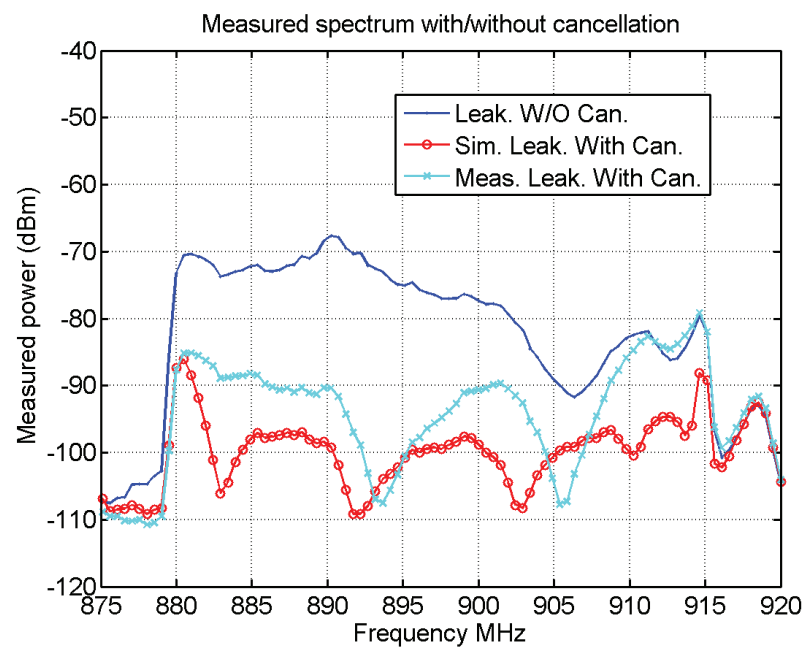

Fig. 4. Measured Leakage Signals with and without the Cancellation System

The degradation of the cancellation performance is mainly due to the mismatch between the estimated and the implemented delays. Another aspect for such performance decrease is from the undesired properties of the phase shifters that are used in the test bench, which include the dependency of the group delay and insertion loss with respect to the control voltage.

Comparing with one of the most recent TX leakage cancellation techniques in [3] (shown in Table I), the bandwidth of the proposed cancellation is significantly larger. Though the measured P-P. performance of the proposed system is not as good as Zhang's technique, it is mainly due to the
TABLE I

PERFORMANCE COMPARISON

\begin{tabular}{|c|c|c|c|}
\hline & Zhang et & \multicolumn{2}{|c|}{ This Work } \\
\cline { 3 - 4 } & al. [3] & Measured & Modelling \\
\hline \hline Technique & FPC & \multicolumn{2}{|c|}{ Tap based } \\
\hline Duplexer type & FBAR & \multicolumn{2}{|c|}{ Ceramic } \\
\hline Freq. (MHz) & 1950 & \multicolumn{2}{|c|}{897.5} \\
\hline BW (MHz) & 5 & \multicolumn{2}{|c|}{35} \\
\hline P-P. Can. (dB) & 23 & 11 & 20 \\
\hline
\end{tabular}

implementation of the system. The simulated result shows that the P-P. Can. is very close to the opponent with a much wider bandwidth and is only limited to the edge of the band.

\section{CONCLUSIONS}

In order to reduce the pressure on the cost and form factor of the FDD transceiver and to relax the design complexity of the duplexer, a digitally assisted RF cancellation system has been presented in this paper. The system has been estimated using the proposed MADF-DC technique and implemented using dedicated and laboratory fabricated components for demonstration purposes. An off-the-shelf ceramic duplexer is used as the duplexing device in the test bench and $11 \mathrm{~dB}$ of peak-to-peak leakage cancellation for $35 \mathrm{MHz}$ bandwidth has been measured. Though the measured result is not as good as the simulated one, better performance can be achieved with more accurate delay lines and higher performance phase shifters (or vector modulators). The simulated result shows that the performance of the proposed cancellation system is comparable to the state-of-the-art technique, but with much wider bandwidth.

\section{ACKNOWLEDGEMENT}

This material is based upon works supported by the Science Foundation Ireland under Grant No. 10/CE/I1853 as part of the Centre for Telecommunications Research (CTVR). The authors gratefully acknowledge this support.

\section{REFERENCES}

[1] P. Pratt, P. Forbes, and J. McCormack, "Digitally assisted rf filter self interference cancellation system," in European Microwave Conference (EuMC), 2014 44th, Oct 2014, pp. 929-932.

[2] T. Huusari, Y.-S. Choi, P. Liikkanen, D. Korpi, S. Talwar, and M. Valkama, "Wideband self-adaptive rf cancellation circuit for fullduplex radio: Operating principle and measurements," in Vehicular Technology Conference (VTC Spring), 2015 IEEE 81st, May 2015, pp. 1-7.

[3] T. Zhang, A. Suvarna, V. Bhagavatula, and J. Rudell, "An integrated cmos passive self-interference mitigation technique for fdd radios," Solid-State Circuits, IEEE Journal of, vol. 50, no. 5, pp. 1176-1188, May 2015.

[4] H. Su, G. Szczepkowski, and R. Farrell, "Wideband tx leakage cancellation using adaptive delay filter at rf frequencies," in Irish Signals Systems Conference 2014 and 2014 China-Ireland International Conference on Information and Communications Technologies (ISSC 2014/CIICT 2014). 25th IET, June 2014, pp. 396-401.

[5] D. Etter, "Identification of sparse impulse response systems using an adaptive delay filter," in Acoustics, Speech, and Signal Processing, IEEE International Conference on ICASSP '85., vol. 10, Apr 1985, pp. 11691172 . 